\title{
Image-Based Quantification of Plant Immunity and Disease
}

\author{
Bradley Laflamme, ${ }^{1}$ Maggie Middleton, ${ }^{2}$ Timothy Lo, ${ }^{1}$ Darrell Desveaux, ${ }^{1,2}$ and David S. Guttman ${ }^{1,2}$ \\ ${ }^{1}$ Department of Cell \& Systems Biology, University of Toronto, Ontario, Canada; and ${ }^{2}$ Centre for the Analysis of Genome \\ Evolution \& Function, University of Toronto
}

Accepted 2 November 2016.

\begin{abstract}
Measuring the extent and severity of disease is a critical component of plant pathology research and crop breeding. Unfortunately, existing visual scoring systems are qualitative, subjective, and the results are difficult to transfer between research groups, while existing quantitative methods can be quite laborious. Here, we present plant immunity and disease image-based quantification (PIDIQ), a quantitative, semi-automated system to rapidly and objectively measure disease symptoms in a biologically relevant context. PIDIQ applies an ImageJ-based macro to plant photos in order to distinguish healthy tissue from tissue that has yellowed due to disease. It can process a directory of images in an automated manner and report the relative ratios of healthy to diseased leaf area, thereby providing a quantitative measure of plant health that can be statistically compared with appropriate controls. We used the Arabidopsis thaliana-Pseudomonas syringae model system to show that PIDIQ is able to identify both enhanced plant health associated with effector-triggered immunity as well as elevated disease symptoms associated with effector-triggered susceptibility. Finally, we show that the quantitative results provided by PIDIQ correspond to those obtained via traditional in planta pathogen growth assays. PIDIQ provides a simple and effective means to nondestructively quantify disease from whole plants and we believe it will be equally effective for monitoring disease on excised leaves and stems.
\end{abstract}

Research in plant-pathogen interactions provides an important tool in the efforts to mitigate pathogen-mediated crop loss. A crucial step in this process is the screening of plant lines to identify genetic variants with enhanced immunity (Christou and Twyman 2004). These screens have traditionally involved infecting large numbers of plants with pathogen inocula by dip, spray, or pressure infiltration. Assessment of disease symptoms is commonly done by scoring visible phenotypes, for instance, the presence of chlorotic tissue, stunted growth, and necrotic lesions. While there are a number of automated systems available to perform these tasks (e.g., LemnaTec), the cost of these systems is outside the budget of most labs, and therefore, most phenotyping is done by eye. Visual scoring of disease and

B. Laflamme and M. Middleton contributed equally and D. Desveaux and D. S. Guttman contributed equally.

Corresponding authors: D. Desveaux; E-mail: darrell.desveaux@utoronto.ca and D. S. Guttman; E-mail: david.guttman@utoronto.ca

*The $\boldsymbol{e}$-Xtra logo stands for "electronic extra" and indicates that five supplementary figures are published online.

@ 2016 The American Phytopathological Society immunity phenotypes has the advantage of being quick, easy, and biologically relevant. On the other hand, visual scoring suffers due to its highly subjective nature and its lack of resolution and quantification. The latter factors can result in an inability to distinguish subtle phenotypes, while the subjective nature of these assessments results in low portability, in the sense of being difficult to standardize between research groups.

Quantitative tests of disease and immunity have been developed and are widely used (Chakravarthy et al. 2009; Lloyd et al. 2014; Nguyen et al. 2010). These assays measure factors such as bacterial growth, expression of genes induced during immunity (Navarro et al. 2004), deposition of callose in the plant cell wall (Adam and Somerville 1996), production of reactive oxygen species (Alvarez et al. 1998), and chlorophyll abundance (Mecey et al. 2011), among others; however, all of these assays are fairly laborious, and most measure proxies for disease rather than directly measuring disease symptoms. A quantitative, luminescence-based assay has also been developed to monitor in planta bacterial populations using Pseudomonas syringae tagged with the Photorhabdus luminescens luxCDABE operon (Fan et al. 2008). While this approach is high-throughput and precise, it requires the destructive sampling of plant tissue to measure luminescence as well as the generation and use of a modified $P$. syringae strain. Other image-based applications have also been developed to assess plant disease symptoms, but all of these are either commercial products, platform-specific, or require leaves to be excised and placed on a high-contrast background (Table 1).

We have developed plant immunity and disease image-based quantification (PIDIQ) to facilitate the rapid and objective quantification of disease symptoms in a biologically relevant context. PIDIQ measures areas of green (i.e., healthy) and chlorotic (i.e., diseased) tissue and compares the ratio of these values within and among treatments, to generate quantitative data with which both bacterial virulence and plant immune response can be assessed. We validated PIDIQ using the Arabidopsis thalianaPseudomonas syringae model system of plant pathology. A crucial virulence mechanism of $P$. syringae is the type III secretion system, which the pathogen uses to inject effector proteins directly into plant cells to circumvent the innate immune response and promote virulence. Plant species such as Arabidopsis have evolved the capability of recognizing certain effectors to induce a robust effector-triggered immune response that renders pathogens avirulent (Jones and Dangl 2006). Since effectors can promote both plant disease and immunity, we have used them to assess the ability of PIDIQ to capture their contribution to the pathogenic outcome.

PIDIQ is semiautomated and highly quantitative and, thereby, allows the identification of subtle differences in disease state that 
closely reflect data from bacterial growth assays. Additionally, PIDIQ is cross-platform, nondestructive, and does not require special preparation, and thus, can be seamlessly incorporated into any lab's existing workflow.

\section{RESULTS AND DISCUSSION}

\section{PIDIQ workflow.}

PIDIQ is an ImageJ macro (Schneider et al. 2012) that quantifies the relative ratio of healthy to diseased (chlorotic) leaf tissue from photographic images (Fig. 1; Supplementary Fig. S1). Plants grown in flats, pots, plates, or any other container can be used and organized based on the desired experimental design and structure. Photographs are taken of each plant, flat, or container, using a standard digital camera and white light. PIDIQ performs its analysis on a photo-by-photo basis, so images of individual plants need to be extracted if a single photo is taken of a flat or container holding multiple plants. This step can be performed using any standard image processing software. We recommend that images from separate replicates and treatments be stored in independent subdirectories. Upon execution, PIDIQ queries the user for the location of the working directories and, then, processes all the images in each directory on a directoryby-directory basis. For each specified directory, PIDIQ creates a new subdirectory containing individual masked image files and a single data file (in text format) consisting of tab-delimited numeric values corresponding to the areas of green and yellow obtained from each image. These values can be easily copied to a summary spreadsheet for the experiment by the user and then used to calculate the percent of yellow (chlorotic) leaf area. We recommend that users arcsine-transform all values prior to statistical analysis to correct for distributional biases. Since PIDIQ is agnostic as to the type of material it processes in each photo, it can be used to assess the percent yellow in individual excised leaves or stems or even entire containers holding multiple plants if desired by the experimenter.

\section{Quantifying immunity with PIDIQ.}

Pseudomonas syringae pv. tomato DC3000 (DC3000) is a commonly studied phytopathogenic bacterium due to its high virulence in the model plant Arabidopsis (Yao et al. 2013). Tissue chlorosis is a typical disease symptom associated with DC3000 infection of Arabidopsis (Katagiri et al. 2002). In contrast, when DC3000 heterologously expresses the type III secreted effector protein HopZ1a, there is a large decrease in virulence due to the elicitation of HopZ1a-mediated effector-triggered immunity (Lewis et al. 2008). We spray-inoculated Arabidopsis plants with DC3000 carrying empty vector (DC3000::EV), wildtype HopZ1a (DC3000::hopZ1a), or the catalytic HopZ1a (DC3000::hopZ1a C/A) mutant. While the DC3000::EV and
DC3000::hopZ1a C/A treatments showed similarly high levels of chlorosis and disease at 3 days postinfiltration, the plants sprayed with DC3000::hopZla remained healthy, due to the strong immune response (Fig. 2A). We quantified the differences among treatments using PIDIQ and found no significant difference in the percent yellowness between the DC3000::EV and DC3000::hopZ1a C/A treatments, while both treatments showed significantly more chlorosis than the DC3000::hopZ1a treatment (Fig. 2B; Supplementary Fig. S2, $P<0.005$, Tukey honestly significant differences (HSD) post hoc test). We validated these results using standard bacterial growth assays, which showed that the increased chlorosis observed with DC3000::EV and DC3000::hopZ1a C/A correlated with increased bacterial growth relative to DC3000::hopZ1a (Fig. 2C; Supplementary Fig. S3, $P<0.005, t$ test).

\section{Quantifying virulence with PIDIQ.}

While DC3000 is an excellent strain for the study of effectormediated immune elicitation, it is not as well-suited for the study of effector-mediated virulence (susceptibility), simply because it is natively highly virulent in Arabidopsis. Therefore, we used $P$. syringae pv. maculicola $\mathrm{M} 6 \Delta \mathrm{E}(\mathrm{M} 6 \Delta \mathrm{E})$, a virulence plasmid-cured mutant of M6 that is also missing a chromosomal HopX gene (Rohmer et al. 2003), to determine if PIDIQ could be used to quantitatively measure enhanced disease symptoms conferred by an effector protein. M6 $\Delta \mathrm{E}$ grows less robustly in Arabidopsis than DC3000 and has been used to test for slight growth advantages conferred by pathogen effectors in syringe-infiltration growth assays (Belkhadir et al. 2004). For this purpose, we heterologously expressed the $P$. syringae type III secreted effector HopZ2, which, in contrast to HopZ1a, does not elicit an Arabidopsis immune response and, instead, has been shown to enhance the in planta growth of $P$. syringae (Lewis et al. 2008). The catalytic mutant of HopZ2 (C/A) does not confer a virulence advantage to $P$. syringae and grows to the same level as an empty vector-carrying strain (Lewis et al. 2008). After spray-inoculation of M6 $\Delta \mathrm{E}$-carrying HopZ2 (M6 $\mathrm{E}$ ::hopZ2) or the HopZ2 catalytic mutant (M6 $\triangle \mathrm{E}:$ :hopZ2 C/A), we observed a subtle increase in chlorotic disease symptoms at 5 days postinfiltration in $\mathrm{M} 6 \Delta \mathrm{E}:$ :hopZ2-treated plants when compared with M6 $\mathrm{E}:$ :hopZ2 C/A treated plants, with both treatments resulting in more chlorosis than $\mathrm{M} 6 \Delta \mathrm{E}$ ::hopZla-treated plants (Fig. 3A). The phenotypic differences between the

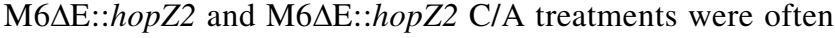
very slight, making it difficult to confidently distinguish between treatments by visual scoring alone. In contrast, PIDIQ identified statistically significant differences between all three treatments in all experimental replicates (Fig. 3B; Supplementary Fig. S4, $P<0.005$, Tukey HSD post hoc test). We again found that the PIDIQ data recapitulated the differences obtained

Table 1. Currently available imaging applications for plant disease quantification

\begin{tabular}{lll}
\hline Software & \multicolumn{1}{c}{ Summary } & Source or citation \\
\hline Assess & Measures total leaf area, lesion count, and disease or damage symptom area. & American Phytopathological Society, \\
& Windows-only. No free license. & St. Paul, MN, U.S.A. \\
BioLeaf & Measures percent damaged leaf area, leaf edge reconstruction. Android-only. & UpVision, Ponta Porã, Brazil \\
CompuEye & Measures total leaf area and disease or damage symptom area. Bin and grid algorithm & Baks \\
& requires high-resolution scans of isolated leaves. Windows-only. & Pethybridge and Nelson 2015 \\
Leaf Doctor & Measures percent diseased leaf area by color data. iOS only. & Green et al. 2012 \\
PhenoPhyte & Measures total leaf area and disease or damage symptom area. Requires physically & Delta-T Devices, London \\
WinDIAS & separated plants for analysis. Requires color and pixel size calibration tool. & \\
& $\begin{array}{l}\text { Measures total leaf area, lesion count, and disease or damage symptom area. Requires } \\
\text { high-resolution scans of isolated leaves on white background. Windows only. No free }\end{array}$ & Whigham et al. 2015 \\
\hline LeafQuant VIGS & license. & Measures percent diseased leaf area using MatLab. Conversion to greyscale discards \\
& valuable color information. Requires user to input leaf count. & \\
\hline
\end{tabular}


from in planta growth assays, and indeed, those differences were more pronounced as scored by PIDIQ than by growth assay (Fig. 3C, $P<0.05, t$ test). Notably, the distinction between the M6 $\mathrm{E}:: h o p Z 2$ and the M6 $\mathrm{E}:: h o p Z 2 \mathrm{C} / \mathrm{A}$ treatments was lower in the growth assay (Fig. 3C; Supplementary Fig. S5) than in the PIDIQ assay (Fig. 3B), which showed strong and consistent differences between these treatments. PIDIQ could also identify significant differences between M6 $\triangle \mathrm{E}:$ :hopZ2 and M6 $\mathrm{E}:$ :hopZ2 C/A treatments as early as 3 days postinfiltration, suggesting that the method has a higher sensitivity to identify subtle differences.

\section{Conclusion.}

Objectively measuring plant health is a critical step in nearly all aspects of fundamental and applied plant pathology research and, unfortunately, there are few methods for scoring disease symptoms or progression that do not rely on subjective disease scores. Here, we present PIDIQ, which provides an objective, rapid, nondestructive, and semiautomated means to quantitatively evaluate the degree of pathogenesis-associated chlorosis. While PIDIQ was developed using the Arabidopsis-P. syringae pathosystem, the inherent flexibility of the method allows adaptation to different plant species, inoculation methods, and propagation methods. The method should also work on excised plant tissue, such as leaves and stem, as well as on field-grown plants. Normalizing results across different experiments using uninfected plants would allow for comparisons between plants grown under significantly different conditions. We believe that PIDIQ can be robustly and easily used in any plant-pathogen system that produces chlorosis or any other change in tissue color (e.g., bleaching, browning) as a symptom of disease.

\section{MATERIALS AND METHODS}

\section{Bacterial strains and constructs.}

We grew Pseudomonas syringae pv. tomato DC3000 and $P$. syringae pv. maculicola M6 $\Delta \mathrm{E}$ in King's medium B (KB) at $30^{\circ} \mathrm{C}$ with rifampicin, which we supplemented with cycloheximide when plating bacteria extracted from plants. Wildtype and catalytic mutants of the two $P$. syringae type III secreted effector genes hopZla and hopZ2 were expressed under their native promoter in frame with a hemagglutinin epitope tag as previously described (Lewis et al. 2008). The immune elicitation activity of HopZ1a is dependent on a catalytic cysteine at position 216, while the virulence-enhancing activity of the HopZ2 allele is dependent on a catalytic cysteine at residue 229. Catalytic (null) mutants for these two effectors have the catalytic cysteine replaced with an alanine (C/A) (Lewis et al. 2008).

\section{Spray infiltration and in planta growth assays.}

We resuspended DC3000 and M6 $\Delta \mathrm{E}$ to an optical density at $600 \mathrm{~nm}\left(\mathrm{OD}_{600}\right)$ of 0.8 in $10 \mathrm{mM} \mathrm{MgSO} 4$ supplemented with $0.04 \%$ Silwet. Prior to inoculation, we serially diluted a $20-\mu 1$ aliquot of each inoculum and plated them on $\mathrm{KB}$ with rifampicin and cycloheximide for colony counting, in order to ensure that there was no significant difference in the concentration of strains across treatments at day 0 (data not shown). We used compressed-air sprayers to spray the strains on four- to fiveweek-old Arabidopsis thaliana ecotype Col-0 plants. Roughly $2.5 \mathrm{ml}$ of $P$. syringae at $\mathrm{OD}_{600}=0.8$ was administered to each plant. We immediately covered the plants with a clear dome after spraying and kept them domed for the remainder of the experiment. For growth assays, we harvested four disks $\left(1 \mathrm{~cm}^{2}\right)$ per plant at day 3 or 4 after spraying, for DC3000-infiltrated plants, and at day 5, for M6 $\Delta \mathrm{E}$-infiltrated plants. These were ground in $10 \mathrm{mM} \mathrm{MgSO}$ and were plated on $\mathrm{KB}$ with rifampicin and cycloheximide for colony counting. Arabidopsis plants were grown with $9 \mathrm{~h}$ of light $\left(130 \mu \mathrm{E} / \mathrm{m}^{2}\right)$ and $15 \mathrm{~h}$ of darkness at $22^{\circ} \mathrm{C}$ in Sungro Professional Growing Mix soil supplemented with $1 \mathrm{~g}$ per liter of 20:20:20 fertilizer. For plants infiltrated with $\mathrm{M} 6 \Delta \mathrm{E}$, we maintained humidity levels below $40 \%$, as higher humidity affected development of chlorotic symptoms.

\section{Image processing and analysis.}

We took all photographs with a Nikon D5200 DSLR camera fitted with a Nikon 18 to $140 \mathrm{~mm}$ DX VR lens under controlled lighting conditions at a fixed height to ensure color consistency and to minimize image distortion. Flats were photographed

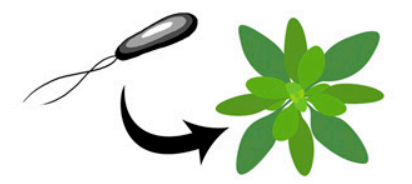

Infiltrate plant tissue with pathogen
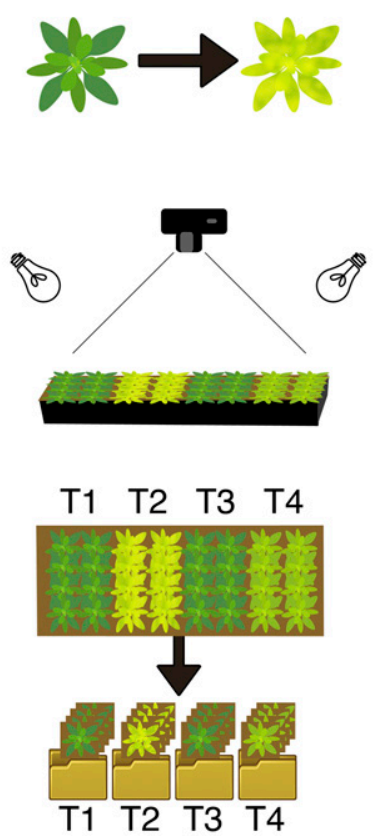

Crop photos to isolate individual plants or treatments
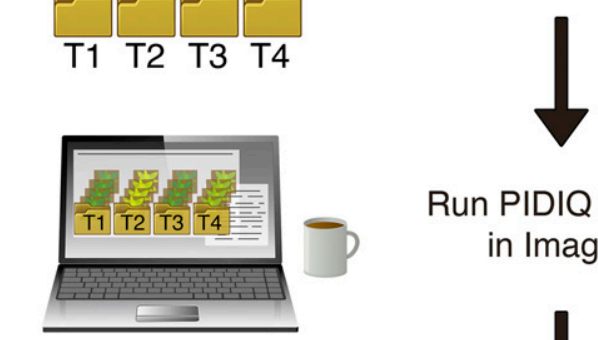

Run PIDIQ macro in ImageJ
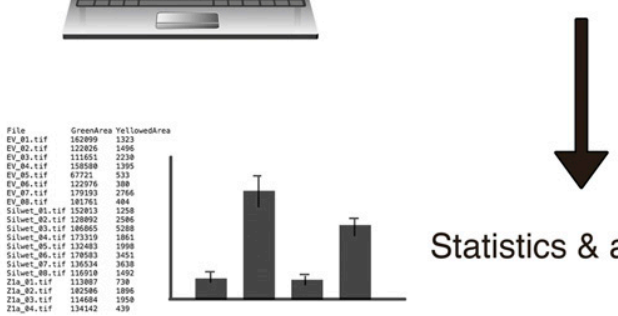

Statistics \& analysis

Fig. 1. Plant immunity and disease image-based quantification (PIDIQ) workflow outline. Plants are infiltrated by dip, spray, or pressure. After symptom development, flats are photographed from directly overhead. Photos should be cropped to isolate treatments for a broad overview or to individual plants for statistically rigorous results. The ImageJ macro function is used to run PIDIQ on the resulting image files and produce a summary text file of healthy and chlorotic tissue areas for each image. The user can then run appropriate statistics (such as analysis of variance) on the data. 
immediately before treatment (day 0), and then, at 24-h intervals until day 3 or 4 for flats treated with DC3000 or until day 5 for flats treated with $\mathrm{M} 6 \Delta \mathrm{E}$.

We used ImageJ (Schneider et al. 2012) to crop each plant individually for greater accuracy and statistical power. We saved the cropped photographs in TIFF format for processing to ensure minimal loss of chroma and pixel detail and ran these images through the PIDIQ macro. The macro, available on Github, automates the image processing steps described below.

For each image, the green and yellow wavelengths are individually masked by ImageJ's color threshold function in HSB mode, using upper and lower bounds for hue and brightness, which we determined empirically on a test set. Default settings for the green spectrum are hue min 50, max 104 and brightness min 43, $\max 255$. For the yellow spectrum, default settings are hue min 33, $\max 50$ and brightness min 134, max 255. The Analyze Particles ImageJ function is used to define masked areas to measure. We set a lower bound for particle size at 50, to omit small specks of algae on vermiculite in the soil, and measured masked green (healthy) and yellow (chlorotic) areas separately per plant in ImageJ. These measurements are then exported to a text file for analysis.

To control for rosette size variation, we determined the proportion of chlorotic to healthy areas for each plant, using the exported measurements. The total proportion of chlorotic to healthy tissue was calculated for each treatment on day 0 and day 3 , for the set treated with DC3000, and on days 0,3 , and 5, for the set treated with $\mathrm{M} 6 \Delta \mathrm{E}$. Raw proportional values were arcsinetransformed and normalized to correct for the distribution bias

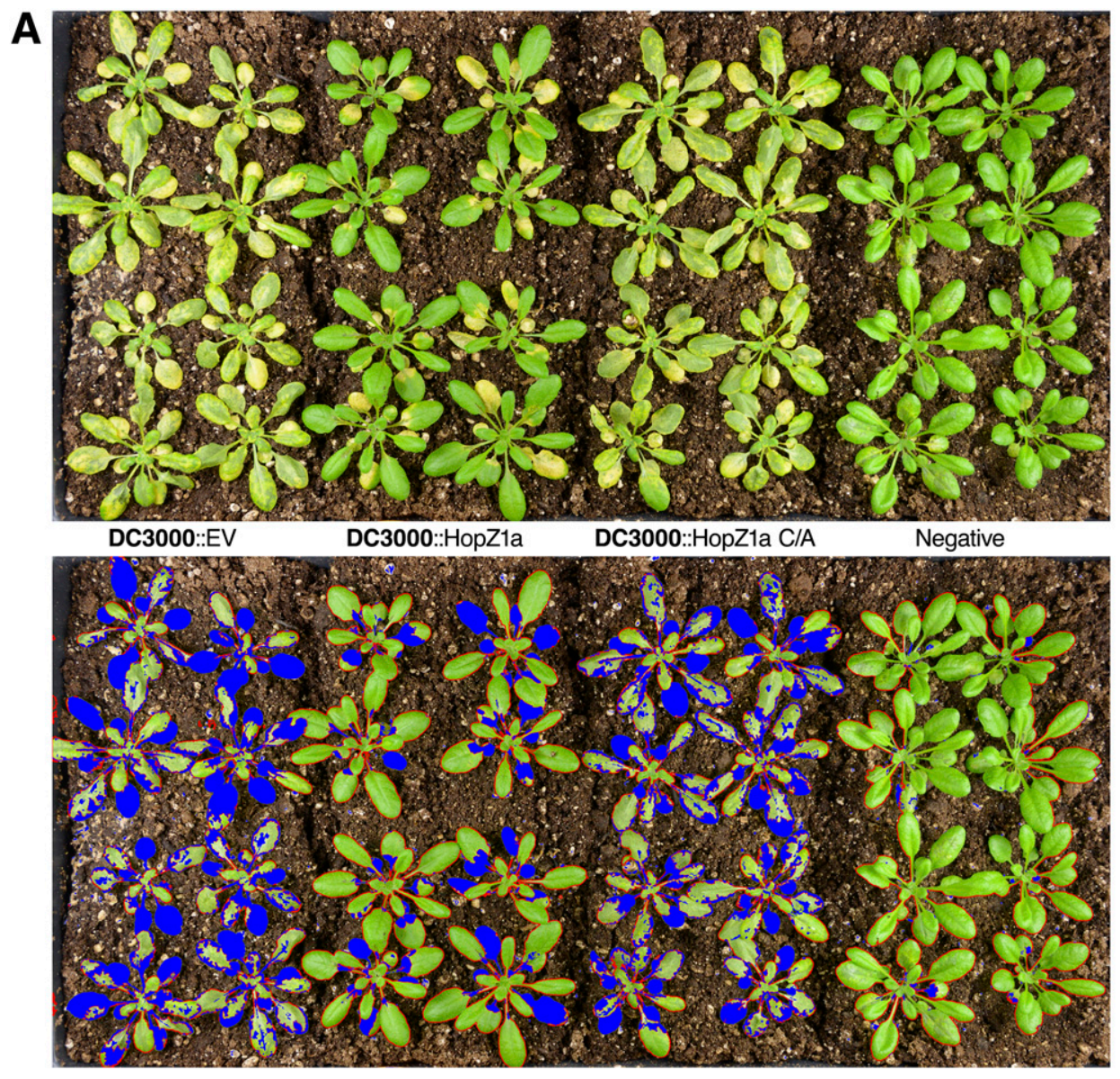

B

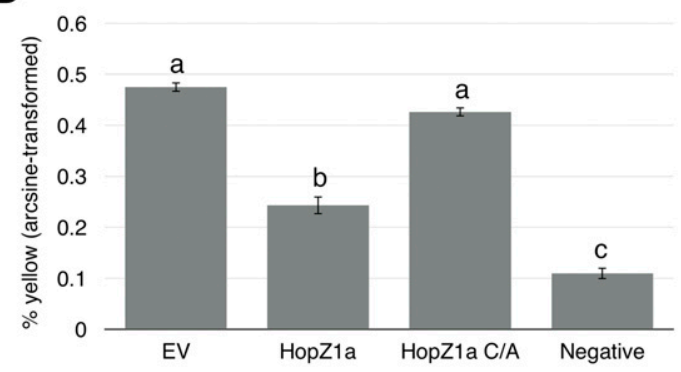

C

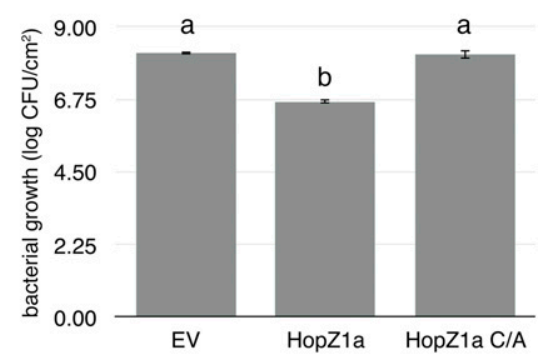

Fig. 2. A, Arabidopsis Col-0 plants infiltrated with Pseudomonas syringae pv. tomato DC3000 carrying the empty vector pUCP20 (EV), HopZ1a (Z1a), catalytic mutant HopZ1a(C/A), and a negative control before (top panel) and after (bottom panel) digital masking in ImageJ using PIDIQ (plant immunity and disease image-based quantification). Healthy regions are outlined in red, chlorotic regions are masked in blue. B, Normalized arcsine-transformed proportion of chlorotic tissue per treatment at 3 days after infiltration ( $n=16$ for each treatment). Pairwise statistical comparisons were performed via Tukey's honestly significant differences post hoc test, with significance groups denoted by letters $(P<0.005)$. Error bars indicate standard error. $\mathbf{C}$, Log-transformed bacterial growth in CFU per square centimeter on day 3 ( $n=8$, for each treatment). Statistical differences were measured between EV and the two HopZ1a treatments via $t$ test $(P<0.005)$. Error bars indicate standard error. 

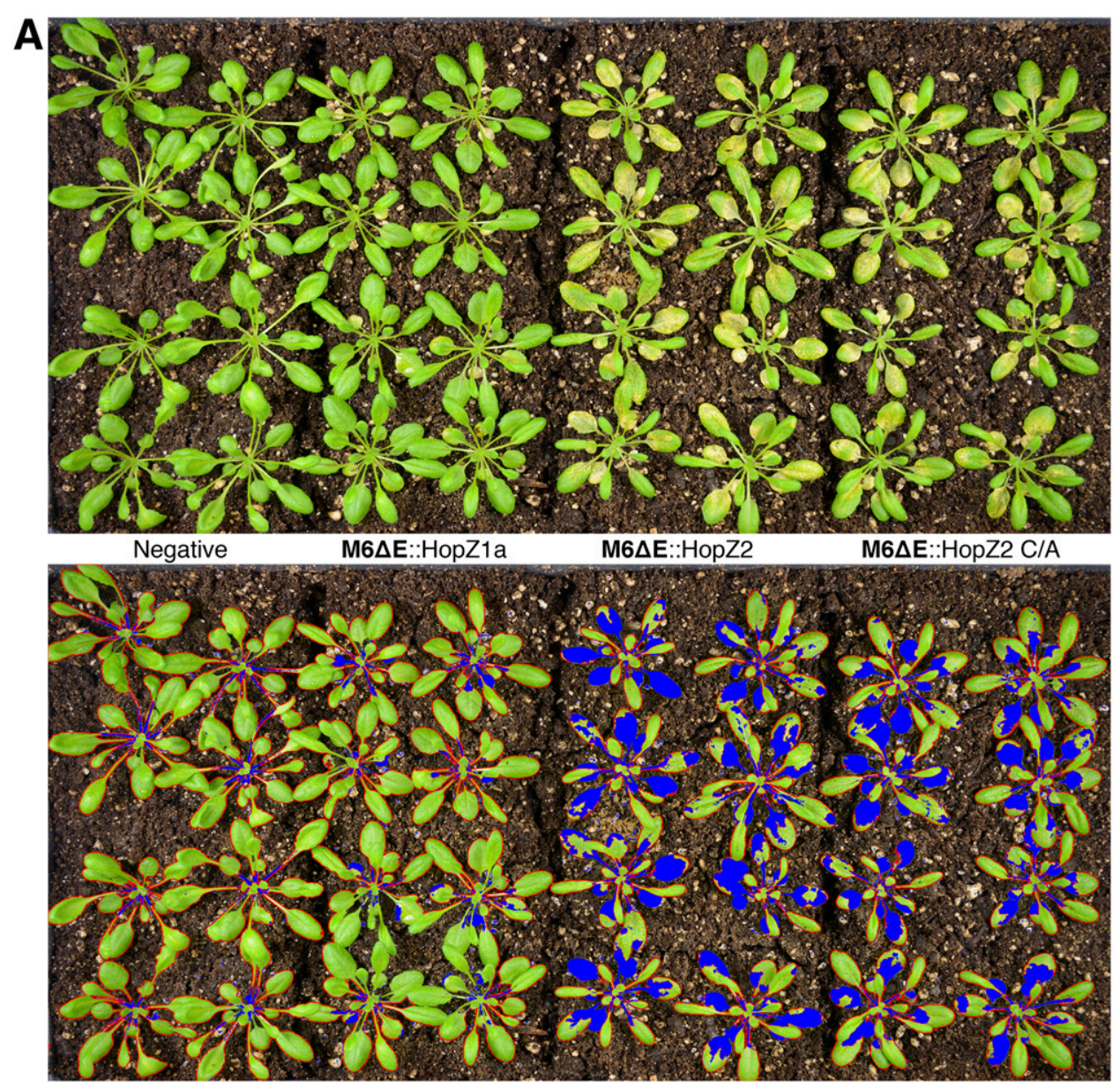

B

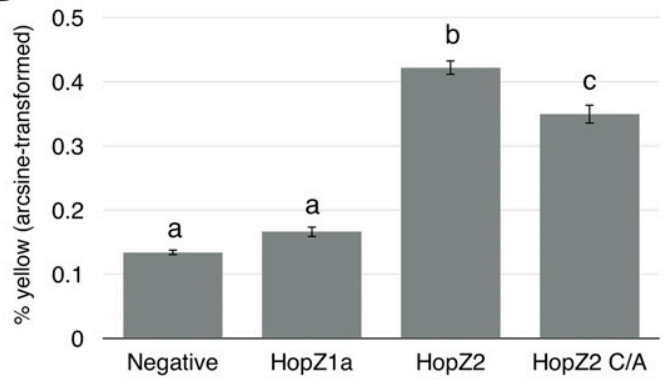

C

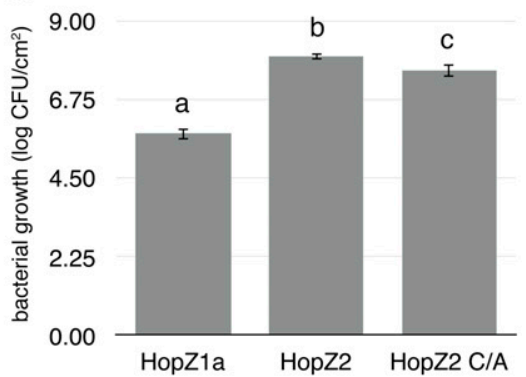

Fig. 3. A, Arabidopsis Col-0 plants untreated or infiltrated with Pseudomonas syringae pv. maculicola M6 $\Delta \mathrm{E}$ carrying HopZ1a, HopZ2, and the catalytic mutant HopZ2(C/A) before (top panel) and after (bottom panel) digital masking in ImageJ, using PIDIQ (plant immunity and disease image-based quantification). Healthy regions are outlined in red, chlorotic regions are masked in blue. B, Normalized arcsine-transformed proportion of chlorotic tissue per treatment at 5 days after infiltration $(n=16$, for each treatment). Pairwise statistical comparisons at day 5 were performed via Tukey's honestly significant differences post hoc test, with significance groups denoted by letters $(P<0.005)$. Error bars indicate standard error. C, Log-transformed bacterial growth in CFU per square centimeter on day 5 ( $n=8$, for each treatment). Statistical differences were measured between EV and the two HopZ2 treatments via $t$ test $(P<$ $0.05)$. Error bars indicate standard error.

associated with bounded proportional data. We performed a one-way analysis of variance with post hoc Tukey's test for each experimental set.

\section{ACKNOWLEDGMENTS}

We thank members of the Guttman and Desveaux labs for valuable input on the project, B. Hurr of Syngenta for development of the macro script, and M. Layeghifard for script debugging. This work was supported by a Natural Sciences and Engineering Research Council of Canada postgraduate award (B. Leflamme), Natural Sciences and Engineering Research Council of Canada Discovery Grants (D. Desveaux and D. S. Guttman); a Canada Research Chair in Plant-Microbe Systems Biology (D. Desveaux) or Compar- ative Genomics (D. S. Guttman), and the Centre for the Analysis of Genome Evolution and Function (D. Desveaux and D. S. Guttman).

\section{LITERATURE CITED}

Adam, L., and Somerville, S. C. 1996. Genetic characterization of five powdery mildew disease resistance loci in Arabidopsis thaliana. Plant J. 9:341-356.

Alvarez, M. E., Pennell, R. I., Meijer, P. J., Ishikawa, A., Dixon, R. A., and Lamb, C. 1998. Reactive oxygen intermediates mediate a systemic signal network in the establishment of plant immunity. Cell 92:773-784

Bakr, E. M. 2005. A new software for measuring leaf area, and area damaged by Tetranychus urticae Koch. J. Appl. Entomol. 129:173-175.

Belkhadir, Y., Nimchuk, Z., Hubert, D. A., Mackey, D., and Dangl, J. L. 2004. Arabidopsis RIN4 negatively regulates disease resistance 
mediated by RPS2 and RPM1 downstream or independent of the NDR1 signal modulator and is not required for the virulence functions of bacterial type III effectors AvrRpt2 or AvrRpm1. Plant Cell 16:2822-2835.

Chakravarthy, S., Velásquez, A. C., and Martin, G. B. 2009. Assay for pathogen-associated molecular pattern (PAMP)-triggered immunity (PTI) in plants. J. Vis. Exp. 31:e1442.

Christou, P., and Twyman, R. M. 2004. The potential of genetically enhanced plants to address food insecurity. Nutr. Res. Rev. 17:23-42.

Fan, J., Crooks, C., and Lamb, C. 2008. High-throughput quantitative luminescence assay of the growth in planta of Pseudomonas syringae chromosomally tagged with Photorhabdus luminescens luxCDABE. Plant J. 53:393-399.

Green, J. M., Appel, H., Rehrig, E. M., Harnsomburana, J., Chang, J.-F., Balint-Kurti, P., and Shyu, C.-R. 2012. PhenoPhyte: A flexible affordable method to quantify 2D phenotypes from imagery. Plant Methods 8:45.

Jones, J. D., and Dangl, J. L. 2006. The plant immune system. Nature 444: 323-329.

Katagiri, F., Thilmony, R., and He, S. Y. 2002. The Arabidopsis thalianaPseudomonas syringae interaction. Arabidopsis Book 1:e0039.

Lewis, J. D., Abada, W., Ma, W., Guttman, D. S., and Desveaux, D. 2008. The HopZ family of Pseudomonas syringae type III effectors require myristoylation for virulence and avirulence functions in Arabidopsis thaliana. J. Bacteriol. 190:2880-2891.

Lloyd, S. R., Schoonbeek, H. J., Trick, M., Zipfel, C., and Ridout, C. J. 2014. Methods to study PAMP-triggered immunity in Brassica species. Mol. Plant-Microbe Interact. 27:286-295.

Mecey, C., Hauck, P., Trapp, M., Pumplin, N., Plovanich, A., Yao, J., and He, S. Y. 2011. A critical role of STAYGREEN/Mendel's I locus in controlling disease symptom development during Pseudomonas syringae pv tomato infection of Arabidopsis. Plant Physiol. 157: 1965-1974.
Navarro, L., Zipfel, C., Rowland, O., Keller, I., Robatzek, S., Boller, T., and Jones, J. D. 2004. The transcriptional innate immune response to flg22. Interplay and overlap with Avr gene-dependent defense responses and bacterial pathogenesis. Plant Physiol. 135:1113-1128.

Nguyen, H. P., Chakravarthy, S., Velásquez, A. C., McLane, H. L., Zeng, L., Nakayashiki, H., Park, D. H., Collmer, A., and Martin, G. B. 2010. Methods to study PAMP-triggered immunity using tomato and Nicotiana benthamiana. Mol. Plant-Microbe Interact. 23:991-999.

Pethybridge, S. J., and Nelson, S. C. 2015. Leaf Doctor: A new portable application for quantifying plant disease severity. Plant Dis. 99:1310-1316.

Rohmer, L., Kjemtrup, S., Marchesini, P., and Dangl, J. L. 2003. Nucleotide sequence, functional characterization and evolution of $\mathrm{pFKN}$, a virulence plasmid in Pseudomonas syringae pathovar maculicola. Mol. Microbiol. 47:1545-1562.

Schneider, C. A., Rasband, W. S., and Eliceiri, K. W. 2012. NIH Image to ImageJ: 25 years of image analysis. Nat. Methods 9:671-675.

Whigham, E., Qi, S., Mistry, D., Surana, P., Xu, R., Fuerst, G., Pliego, C., Bindschedler, L. V., Spanu, P. D., Dickerson, J. A., Innes, R. W., Nettleton, D., Bogdanove, A. J., and Wise, R. P. 2015. Broadly conserved fungal effector BEC1019 suppresses host cell death and enhances pathogen virulence in powdery mildew of barley (Hordeum vulgare L.) Mol. Plant-Microbe Interact. 28:968-983.

Yao, J., Withers, J., and He, S. Y. 2013. Pseudomonas syringae infection assays in Arabidopsis. Methods Mol. Biol. 1011:63-81.

\section{AUTHOR-RECOMMENDED INTERNET RESOURCES}

Assess 2.0: Image analysis software for plant disease quantification: http://www.scientificsocieties.org/aps/apspress/assess

Github DSGlab/PIDIQ.ijm script: https://gist.github.com/DSGlab/1b3a226a7af884efd9356ea2d6a02bd4 